\title{
Changes in the extracellular matrix during myocardial remodelling
}

\author{
Markéta Hegarová MD, Ivan Málek MD PhD, Josef Kautzner MD PhD
}

\begin{abstract}
M Hegarová, I Málek, J Kautzner. Changes in the extracellular matrix during myocardial remodelling. Curr Res Cardiol 2015;2(1):35-39.

The present article focuses on the pathophysiology of myocardial extracellular matrix (ECM) remodelling in chronic heart failure (CHF). The trigger mechanisms for ECM remodelling include acute loss of contractile elements as a consequence of myocardial ischaemia, mechanical tension, oxidative stress and neurohumoral activation. The ECM phenotype changes as a result of the expression of fetal remodelling genes with the aim of accomplishing reparatory changes. This leads to a change in the composition of the ECM, rearrangement of the existing structures and an alteration of the proportion of individual components. While the ECM
\end{abstract}

espite advances in diagnostics and treatment, chronic heart failure $(\mathrm{CHF})$ remains an important medical and social problem. Understanding of the underlying pathophysiology of CHF has led to the introduction of effective pharmacological and nonpharmacological therapies. However, all treatment strategies that positively impact quality of life and prognosis in CHF affect myocardial remodelling. Myocardial remodelling is a complex process that affects more than just cardiomyocytes. Changes in composition and function of the extracellular matrix (ECM) and nonmyocyte cells also play an important role. The present review focuses on the pathophysiology of ECM changes as part of the CHF syndrome.

\section{PATHOPHYSIOLOGY OF MYOCARDIAL REMODELLING IN CHF}

CHF is most often induced by an initial insult that affects cardiac function. The resulting drop in cardiac output leads to inadequate filling of arterial circulation; subsequently, activation of peripheral receptors results in a series of neurohumoral adaptations. The hallmark of this process is activation of sympathoadrenal and renin-angiotensinaldosterone (RAAS) systems. Other adaptations include nonosmotic release of vasopressin and cytokines. The above change in autonomic balance leads to systemic vasoconstriction that, together with sodium and fluid retention, aims to maintain sufficient perfusion of vital organs. Decreased renal perfusion correlates with reduced cardiac output and leads to a decrease in glomerular filtration and further activation of RAAS. Salt and water retention lead to an increase in preload, and a predominance of potent vasoconstrictors increases afterload. The adaptation mechanisms active at the level of the heart and helping to preserve its pumping function lead to increased heart rate, strength and frequency of contractions with a rise in the preload thanks to the Frank-Starling mechanism, ie, an extension of sarcomeres with a subsequent increase in contraction strength and an expansion of contractile elements.

These ancient phylogenetic compensatory mechanisms already play a role in the acute phase of myocardial damage and represent a salvage process to maintain circulation integrity in situations of inadequate represents the target structure, at the same time, individual ECM components participate to various degrees directly in myocardial remodelling as modulators of a number of signalling pathways. Matricellular proteins are expressed in reaction to pathological stimuli. These have no structural function but modulate interactions between ECM components, nonmyocyte cells and cardiomyocytes. The principal consequence of ECM remodelling is an increase in the fibrous tissue content of the heart. Pharmacological intervention affecting the process of ECM remodelling could improve the prognosis of patients with CHF.

Key Words: Extracellular matrix; Heart failure; Myocardial remodelling

arterial filling. However, long-term activation of these mechanisms soon becomes counterproductive, and heart failure becomes chronic; within a period of weeks to months, the action of neurohumoral activators leads to ventricular remodelling.

The term 'remodelling' denotes the structural changes of the myocardium that represent a reaction to overload of various types. These changes develop independently of the type of primary damage and play an important part in disease progression. Macroscopically, remodelling results in development of some degree of myocardial hypertrophy (primary pressure overload) with subsequent dilation. These adaptations are most pronounced in the left ventricle, which adopts a spherical shape with wall thinning and development of secondary mitral regurgitation. Initially, left ventricular hypertrophy, or that associated with left ventricular dilation, helps to increase contractility, but this benefit is eventually exhausted. The decreased oxygen supply to hypertrophic myocardium leads to an increased susceptibility to arrhythmias and to further structural changes, namely, the loss of physiological ventricular shape.

On the ultrastructural level, the changes that occur are best characterized by the fetal gene program of myocardial restructuring (1). Postnatally, cardiomyocytes are believed to be terminally differentiated cells incapable of further division and any loss of such cells is considered irreversible. However, their cellular content is renewed regularly. A complex of signalling pathways is triggered in cardiomyocytes in reaction to overload, oxidative stress, inflammation and circulating neurohormones. These pathways result in changes in gene expression, post-translation changes and changes in protein synthesis. This process consequently leads to hypertrophy of the cardiomyocytes or to their loss, changes in the structure of contractile proteins, changes in the membrane transport mechanisms and calcium ion compartmentalization, or changes in cell energetics. Myocardial remodelling also involves the ECM and nonmyocyte cells. It is important to note that these structures do not undergo merely 'passive' changes in reaction to neurohumoral activation; on the contrary, they actively participate in all stages of remodelling. Therefore, logically, they become potential targets of new therapeutic interventions.

Institute for Clinical and Experimental Medicine, Cardiology Department, Prague, Czech Republic

Correspondence: Dr Markéta Hegarová, Department of Cardiology, Institute for Clinical and Experimental Medicine, Vídeňská 1958/9, 140 21 Prague 4 ,

Czech Republic. Telephone 420-236055262,fax 420-261362986, e-mail mahg@medicon.cz 


\section{COMPOSITION AND FUNCTION OF THE ECM AND THE ROLE OF MYOFIBROBLASTS IN THE MYOCARDIUM}

Although cardiomyocytes comprise most of the myocardial volume, the actual number of nonmyocyte cells within the myocardium is much higher. More than two-thirds of cells within the cardiac muscle consist of endothelial cells, fibroblasts and pericytes. Macrophages and mastocytes (mast cells) are represented to a smaller extent in the interstitial space. These cellular elements are integrated within the complex ECM network.

The ECM is an important structure consisting of elements that not only provide structural support to the cardiomyocytes and vessels, but also play an important role in the regulation of myocardial contraction and relaxation; they also participate in maintaining homeostasis. These functions are mediated through interaction of ECM components with integrins and other cardiomyocyte cell receptors. The relationship between ECM proteins and nonmyocyte cells (cell-matrix interactions) affects adhesion, cellular survival, proliferation, differentiation and function. The ECM provides protection to fibroblasts against the effects of mechanical stress and, thus, promotes their resting phenotype (2). Viewed complexly, the ECM is essential for maintaining the normal geometry and function of the myocardium.

The ECM components are also of key importance for preserving myocardial function under pathological conditions. A number of ECM proteins bind growth factors released during myocardial damage. Moreover, the products of ECM degradation subsequent to myocardial damage - ECM protein fragments - bind receptors for various growth factors and activate many signalling pathways. The interaction between the ECM and cells represents the essential moderator of adaptive and reparative changes of the myocardium as a reaction to various types of damage. This is enabled by a change in the cell phenotype as well as changes in the number, proportion and composition of ECM proteins.

The ECM is primarily composed of type I fibrillar collagen and, to a lesser extent, collagen III, V, fibronectin and basement membrane proteins (collagen IV, laminin, nidogen-2) and hydrophilic proteoglycans. Proteoglycans are gigantic gel-forming molecules that fill the intercellular space, bind water, increase resistance to pressure and ensure that tissues return to their original shapes. They bind to fibrillar collagen and are capable of binding signalling molecules (eg, fibroblast growth factor). Fibrillar collagen type I and III secreted by myofibroblasts is essential for maintaining myocardial architecture, stabilizing myocytes, and maintaining their shape and spatial arrangement in parallel and series, and in layers. These collagens are responsible for transmitting the force produced by cardiomyocytes during systole. The energy stored in the coiled collagen during systole plays a role in the elongation of myocytes during relaxation and participates in the early filling phase. It is collagen traction force and its resistence that, among others, prevent the rupture of myocardial tissue following myocardial infarction.

Collagen XV represents a special component of the ECM. This collagen has very specific, complex spatial structure $(3,4)$ and is essential for appropriate arrangement of cardiac muscle structures. This collagen, which is flanked by globular domains with attached proteoglycans, is capable of binding microfilaments as well as proteins and proteoglycans of basement membranes. It forms a bridge between the fibrillar ECM components and the basement membranes of myocardial cells and is capable of absorbing mechanical stress and enhancing myocardial mechanical tension resistance (2). Its C-terminus (restin) also exerts a negative effect on angiogenesis (5). In experiments, collagen XV deficiency was associated with impaired contractile response to stimulation with $\beta$ agonists (6); increased capillary permeability; interstitial deposition of nonfibrillar protein aggregates; increased myocardial rigidity; and irregular arrangement of cardiomyocytes. This phenotype predisposes the myocardium to pathological reactivity as a result of stress (7). Other ECM proteins modulating the interaction between the matrix and cells are expressed during embryonic development. These matricellular proteins are rarely expressed in adulthood under physiological conditions. However, they may be detected as part of the fetal program of myocardium remodelling in reaction to various types of damage.

Myofibroblasts represent long-term persisting phenotype-transformed fibroblasts with preserved proliferative potential (in contrast to cardiomyocytes). Myofibroblasts transfer contractile strength, produce ECM components, and have both autocrine and paracrine secretory functions. Their activity and resulting collagen turnover within the myocardium are mainly regulated by products of the RAAS system and fibrogenic cytokines. The content of contractile proteins, the arrangement of myofibroblasts and the proliferative response vary depending on humoral stimulation and mechanical conditions. Myofibroblasts produce collagen type I, III and VI. Collagen type IV is produced by cardiomyocytes and forms part of their basement membrane.

\section{MYOFIBROBLAST ACTIVITY AND ECM CHANGES DURING THE COURSE OF CHF}

Changes in ECM are characteristic of myocardial remodelling in reaction to various types of damage. Alterations of ECM composition occur, together with rearrangement of existing structures and changes in the proportion of individual components, in reaction to neurohumoral activation. At the same time, individual ECM components participate directly to a various extent in myocardial remodelling as modulators of a number of signalling pathways.

The most important process in the course of myocardial remodelling involves the increasing proportion of connective tissue. This is due to stimulation of fibroblast differentiation into myofibroblasts and to an imbalance in the synthesis and degradation of ECM components and their deposition. These developments reflect disrupted equilibrium between the activity of matrix metalloproteinases (MMPs) and their tissue inhibitors (TIMPs). Fibroblast proliferation and increased connective tissue production lead to disconnection of cardiomyocytes (8) and decreased capillary density with impaired oxygen diffusion (9). The higher amount of collagen leads to decreased compliance of the remodelled myocardium, impairment of the diastolic function and increased susceptibility to arrhythmias. Degradation of myocardial collagen leads to a decrease in myocardium rigidity and dilation.

The principal stimuli for myofibroblast differentiation and secretory activity are the products of neurohumoral activation (transforming growth factor $\beta$ (TGF- $\beta$ ), angiotensin (ANG) II, aldosterone, endothelin-1 and tumour necrosis factor- $\alpha$ (TNF $\alpha$ ). The subsequent state and degree of myocardial fibrosis depend on the type of damage incurred and the duration of neurohumoral activation. ANG II is the fundamental mediator of myofibroblast proliferation and ECM production in the failing myocardium. It also increases the production of TGF $\beta$, which promotes collagen synthesis. Together with aldosterone, it activates not only fibroblasts, but also participates in the initiation of cytokine production and chemotaxis of inflammatory elements. ANG II increases the activity of plasminogen I inhibitor, which leads to a decrease in MMP activity during the phase of ventricle dilation.

The ECM of the failing myocardium contains matricellular proteins, macromolecules that lack any structural function, but which interact with cell receptors, growth factors and proteases. In reaction to external stimuli, matricellular proteins affect the behaviour of cells, including cardiomyocytes, and affect changes in the ECM of failing myocardium. Matricellular proteins play a key role in the embryonic period. In CHF, their role changes as they no longer modulate organogenesis and growth, but rather modulate reparative changes. The changes in ECM composition lead directly to a change in the cell phenotype, which falls within the concept of fetal rearrangement. In reaction to loss of tissue due to ischemic necrosis, matricellular proteins modulate changes in matrix organization and migration, proliferation, adhesion and metabolism of cells. During volume overload, matricellular proteins may modulate cytokines and growth factors that affect myocyte hypertrophy, ECM structure as well as the activity of 
fibroblasts and inflammatory cells. The currently known matricellular proteins include tenascin- $\mathrm{C}$, tenascin-X, osteonectin, osteopontin, thrombospondins SPARC (secreted protein acidic and rich in cysteine), periostin and hevin. Proteins that demonstrate certain matricellular properties include fibulin-5, galectins, plasminogen I inhibitor and others (10).

Among the proteins with certain matricellular activities, we include galectin-3, a member of the galectin family. This mediator of inflammatory response and fibrotization is necessary for the phagocytic activity of macrophages. Its expression in the healthy human myocardium is low and increases under pathological conditions. Galectin-3 secreted into the extracellular space has a stimulatory effect on macrophage migration and fibroblast proliferation. It promotes the secretory activity of myofibroblasts and increases production of type I collagen. Via TIMPs, galectin-3 also affects the degree of collagen degradation.

MMPs are a group of zinc-dependent proteases that influence the composition of the basement membranes and ECM by degrading some of its components in reaction to various types of damage. MMPs are synthetized as inactive zymogenes and are most frequently secreted in the form of a pro-enzyme into the ECM, where they bind to a specific protein. MMPs are activated by the cleavage of their pro-domain by proteases, including another serine protease, plasmin, thrombin and certain MMPs. Exposure of their catalytic site may occur even though the action of reactive oxygen species (ROS), alkylating substances, heavy metals and disulfides. Increased MMPs activity is also detectable in drug addicts. MMPs are regulated by endogenous inhibitors - TIMPs. TIMPs are low-molecular proteins capable of binding to the catalytic domain of the active MMP, which prevents the binding of substrate.

\section{THE INFLAMMATORY RESPONSE AND CHANGES IN THE ECM}

Inflammation affects myocardial remodelling as the principal pathophysiological mechanism in the case of myocarditis and inflammatory cardiomyopathy. The trigger mechanism most often involves infection by cardiotropic viruses from the enterovirus and adenovirus families. In the acute phase of the disease, affected cells express on their surface viral antigens together with the antigens of the major histocompatibility complex (HLA system) and adhesive molecules such as intercellular adhesion molecule-1 and lymphocyte function related antigen 3 . They present these to the immune system. At first, $\mathrm{T}$ lymphocytes play a major role in the subsequent inflammatory response. Massive infiltration by these cells is associated either diffusely or focally in the acute phase with the most severe myocardial damage.

Fortunately, the course of acute myocarditis is usually asymptomatic and most cases heal spontaneously. The destruction of cells by viral proteases or the immune reaction only sporadically lead to irreversible loss of myocardial contractility and progressive acute heart failure. In the majority of cases, however, this mechanism is not involved in the etiopathogenesis of myocarditis. Therefore, only a transitory worsening of ventricular systolic function can be observed. Occasionally, systolic dysfunction persists for several months after resolution of acute infection. Once contemporary drug treatment of heart failure is introduced, reverse remodelling (and often normalization of the myocardial systolic function) may be induced. It is probable that in such cases, the disease is not provoked by immune reaction of the organism but rather by the neurohumoral activation induced by the significant decrease in cardiac output at onset of the disease. Inflammatory cardiomyopathy as such is only caused by virus-induced autoimmune damage of the myocardium.

However, the inflammatory activation participates to a certain extent in the process of myocardial remodelling in every case, regardless of the primary cause of the heart failure syndrome. The key mechanism is the injury-triggered activation of innate immunity via the stimulation of toll-like receptors (TLR). Members of the TLR family are responsible for recognizing structures typical of pathogenic molecules (pathogen-associated molecular patterns). These form part of the innate ability of the immune system to recognize foreign pathogens. In the normal human myocardium, expression of TLR-4 is diffuse and predominantly cytoplasmic within cardiomyocytes. Loci of intensive staining of several juxtaposed myocytes have been detected in patients diagnosed with dilated cardiomyopathy (11). Increased TLR-4 expression has also been detected in the myocardium of patients who have had a mechanical circulatory support implanted in the terminal phase of heart failure (12). In the case of myocardial ischemia, TLRs activate ROS and intracellular proteins released from necrotic cells, pathogens as well as heat shock proteins.

TLRs activate the nuclear factor- $\kappa \mathrm{B}(\mathrm{NF}-\kappa \mathrm{B})$ (redox-sensitive transcription factor) signalling pathway, which affects the expression of cytokines via adaptor molecules. Activation of NF- $\mathrm{kB}$ links the innate and adaptive components of the immune response via proinflammatory cytokines and chemokines, as well as by inducing the expression of costimulatory molecules such as CD80, CD86 and CD40. By activating the caspase cascade, the NF- $\mathrm{kB}$ signalling pathway induces apoptosis. The cytokines produced (especially TNF- $\alpha$ and interleukin [IL]-1 $\beta$ ) activate MMPs and participate in the process of ECM degradation. Collagen fragments then amplify the inflammatory response by stimulating not only mononuclear cells but also neutrophils. Neutrophils exacerbate oxidative stress and, by releasing proteases, they further contribute towards collagen degradation. The TGF- $\beta$ released from inflammatory cells stimulates the transdifferentiation of fibroblasts into myofibroblasts. TGF- $\beta$ production is also stimulated by ANG II, endothelin and aldosterone. ANG II also activates the NF- $\mathrm{kB}$ signalling pathway. NF- $\mathrm{kB}$ has a protective effect in hypoxia and re-perfusion damage. Its prolonged activation is harmful, inducing a 'chronic inflammatory state' with the production of TNF $\alpha$, IL- 1 and IL- 6 .

\section{CHANGES IN ECM COMPOSITION AS A CONSEQUENCE OF MYOCARDIAL ISCHEMIA}

During the course of myocardial infarction and the subsequent healing process, the most dynamic changes occur in the myocardial ECM. These adaptations may provide a model situation. The cytoplasmic components released from the dying cells during myocardial infarction activate complement, and ECM fragments together with ROS interact with TLR. This leads to the activation of the NF- $\kappa B$ signalling pathways with up-regulation of cytokines and chemokines (13), triggering the inflammatory response (14). ROS produced as part of oxidative stress during ischemia or reperfusion (15) may directly damage DNA, proteins and cell membranes. These products are also capable of activating the inflammatory response, initiating cardiomyocyte apoptosis, cell hypertrophy and proliferation, as well as fibrogenesis. The principal cause of excessive ROS production in the infarcted myocardium is the NADPH oxidase expressed by neutrophils and macrophages (16). In the infarction border zone, the principal reasons for the high ROS concentration are the increased mitochondrial production of ROS (17) and the intracellular oxidation systems-NADPH oxidase, xanthine oxidase and nitric oxide synthase (18). The necrotic region is often infiltrated by inflammatory cells, especially by neutrophils and monocytes/macrophages. The inflammatory cells are directed to the site of necrosis due to the adhesive molecules and cytokines (chemo-attractants) expressed by the endothelial cells of the coronary vessels in the infarction border zone. During the inflammatory phase, there is very early activation (19) that involves predominantly latent MMPs that degrade the ECM. Synthesis of new MMPs is subsequently induced. The lowmolecular fragments of the degraded ECM components further promote proinflammatory activity. The gradually degraded original matrix is replaced by a provisional network consisting predominantly of fibrin and plasma fibronectin. The migrating mesenchymal cells interact via integrins with ECM molecules, which modulate their gene expression and change of phenotype. Here, an important role is probably played by certain matricellular proteins. Apart from their 
effect on fibroblast phenotype, certain matricellular molecules also play an important role in so-called de-adhesion, ie, the freeing of cells and their subsequent ability to migrate into repaired sites (10). The granulation tissue gradually dissolves the provisional matrix (composed of plasma components). This is followed by rapid building of provisional tissue from cell products - predominantly fibronectin and hyaluronate. Matricellular proteins activate signalling pathways with the aim of forming the final scar, which will ensure the structural integrity of the tissue. By the end of the first week, the proteolytic activity of MMPs has already subsided due to increased expression of TIMPs (20). The inflammatory infiltration culminates in the second week following the coronary event and the inflammatory cells disappear within a month via programmed death. Shortly after the inflammatory elements, myofibroblasts enter the necrotic region. These elements are characterized by the expression of the $\alpha$-smooth muscle actin microfilaments, which ensure their contractility (21). Thanks to their ability to interconnect via the gap junction, myofibroblasts are able to form an elastic network to promote tissue integrity. The macrophage-produced TGF- $\beta$ stimulates the transformation of fibroblasts into the myofibroblast phenotype and initiates their proliferative and secretory activities. This process is modulated by matricellular proteins (10). Apart from the ECM components, myofibroblasts are also capable of expressing renin, angiotensin-converting enzyme (ACE), angiotensinogen and also ANG II receptors (22-24). Thus, together with macrophages, myofibroblasts represent an important activator of local RAAS. Thanks to their ARB expression, they are at the same time one of the principal executors of neurohumoral stimulatory effects.

The initial phase of ECM degradation is followed by the process of stable scar development. Matricellular proteins are removed and polymerisation of the deposited collagen ensues. The scar helps maintain integrity of the ventricle but changes the mechanical properties of the myocardium. However, formation of a localized scar does not represent the end of the fibrotic process by far. The myofibroblasts activated by the neurohumoral response produce connective tissue diffusely within the myocardium. Thus, in the tissue remodelled as a consequence of a coronary event, we find diffuse perivascular fibrosis, fibrotization of arterial and arteriolar walls as well as of the interstitial space. Myofibroblast MMPs participate in the process of myocardial dilation following myocardial infarction.

\section{CLINICAL ASPECTS OF ECM REMODELLING}

The first important clinical aspect in relation to ECM remodelling is estimation of prognosis. The degree of fibrotization may be effectively assessed noninvasively with the aid of late gadolinium enhancement magnetic resonance imaging (25). The incidence of late gadolinium enhancement correlates well with the prognosis of patients with $\mathrm{CHF}$ (26).

\section{REFERENCES}

1. Kuwahara K, Nakao K. New molecular mechanism for cardiovascular disease: Transcriptional pathways and novel therapeutic targets in heart failure. J Pharmacol Sci 2011;116:337-42.

2. Tomasek JJ, Gabbiani G, Hinz B, et al. Myofibroblasts and mechano-regulation of connective tissue remodelling. Nat Rev Mol Cell Biol 2002;3:349-63.

3. Hurskainen M, Ruggiero F, Hägg P, et al. Recombinant human collagen XV regulates cell adhesion and migration. J Biol Chem 2010;285:5258-65.

4. Myers JC, Amenta PS, Dion AS, et al. The molecular structure of human tissue type $\mathrm{XV}$ presents a unique conformation among the collagens. Biochem J 2007;404:535-44.

5. Ramchandran R, Dhanabal M, Volk R, et al. Antiangiogenic activity of restin, NC10 domain of human collagen XV: Comparison to endostatin. Biochem Biophys Res Commun 1999;255:735-9.

6. Eklund L, Piuhola J, Komulainen J, et al. Lack of type XV collagen causes a skeletal myopathy and cardiovascular defects in mice. Proc Natl Acad Sci U S A 2001;98:1194-9.
Among the existing or potential biomarkers, galectin-3 (a protein with matricellular properties) is currently being studied. Its level appears to correlate with the degree of fibrotization and overall mortality in patients with CHF (27), even in cases of very advanced disease (28). In view of the clear association with the degree of fibrotic changes, it might prove useful for estimating the risk of sudden death. For instance, in patients with myocardial remodelling due to pressure overload associated with aortic valve stenosis, determination of galectin-3 and other matricellular proteins together with magnetic resonance imaging could help to identify asymptomatic patients who are at high risk. Another potential application would be identification of high-risk patients prone to develop heart failure. This could result in prophylactic therapy. Other matricellular proteins such as tenascin could prove to be suitable biomarkers for the timely prediction of myocardial remodelling (29).

Another interesting clinical option is the diagnosis of an on-going inflammatory process within the myocardium with an scintigraphy using labelled antibodies against specific matricellular proteins (30).

\section{CONCLUSIONS}

Better understanding the role of individual participants in the process of ECM remodelling will probably result not only in establishing clinically applicable biomarkers, but also in development of new pharmacological strategies. All the therapeutic procedures approved and recommended to date in heart failure influence the pathophysiology of myocardial remodelling and induce reverse remodelling. Drugs that demonstrably affect ECM remodelling and modify the proliferative and secretory activity of myofibroblasts include inhibitors of the RAAs system. Despite combined treatment with ACE inhibitors and blockers of ANG II type 1 receptors together with certain aldosterone blockers (spironolactone or eplerenone) and beta blockers, the prognosis of some patients with CHF remains poor. Therapeutic intervention affecting the interactions between the ECM components, nonmyocardial cells and cardiomyocytes appears to be another promising option. To be more specific, inhibition of galectin-3 could be used to prevent and treat myocardial fibrotization and/or galectin-1 may exert its protective effect on the attenuation of the inflammatory reaction following myocardial infarction (31).

The question remains whether any therapeutic modifications of the signalling pathways of matricellular proteins would, in contrast to, for example, treatment with the neutral endopeptidase inhibitor and ACE omapatrilat, have truly an additive effect to current pharmacotherapy. Another problem would be the optimal timing of potential therapy in view of the highly different and varied processes of ECM remodelling that dominate during the various stages of the disease.

7. Rasi K, Piuhola J, Czabanka M, et al. Collagen XV is necessary for modeling of the extracellular matrix and its deficiency predisposes to cardiomyopathy. Circ Res 2010;107:1241-52.

8. Gaudesius G, Miragoli M, Thomas SP, et al. Coupling of cardiac electrical activity over extended distances by fibroblasts of cardiac origin. Circ Res 2003;93:421-8.

9. Sabbah HN, Sharov VG, Lesch M, Goldstein S. Progression of heart failure: A role for interstitial fibrosis. Mol Cell Biochem 1995;147:29-34.

10. Frangogiannis NG. Matricellular proteins in cardiac adaptation and disease. Physiol Rev 2012;92:635-88.

11. Frantz S, Kobzik L, Kim YD, et al. Toll4 (TLR4) expression incardiac myocytes in normal and failing myocardium. J Clin Invest 1999;104:271-80.

12. Birks EJ, Felkin LE, Banner NR, et al. Increased toll-like receptor 4 in the myocardium of patients requiring left ventricular assist devices. J Heart Lung Transplant 2004;23:228-35.

13. Bujak M, Dobaczewski M, Gonzalez-Quesada C, et al. Induction of the CXC chemokine interferon-gamma-inducible protein 10 
regulates the reparative response following myocardial infarction. Circ Res 2009;105:973-83.

14. Bujak M, Dobaczewski M, Chatila K, et al. Interleukin-1 receptor type I signaling critically regulates infarct healing and cardiac remodeling. Am J Pathol 2008;173:57-67.

15. Siwik DA, Colucci WS. Regulation of matrix metalloproteinases by cytokines and reactive oxygen/nitrogen species in the myocardium. Heart Fail Rev 2004;9:43-51.

16. Fukui T, Yoshiyama M, Hanatani A, et al. Expression of p22-phox angp91-phox, essential components of NADPH oxidase, increases after myocardial infarction. Biochem Biophys Res Commun 2001;281:1200-6

17. Ide T, Tsutsui H, Kinugawa S, et al. Mitochondrial electron transport complex I is a potential source of oxygen free radicals in the failing myocardium. Circ Res 1999;85:357-63.

18. Grieve DJ, Byrne JA, Cave AC, et al. Role of oxidative stress in cardiac remodeling after myocardial infarction. Heart Lung Circ 2004;13:132-8

19. Etoh T, Joffs C, Deschamps AM, et al Myocardial and interstitial matrix metalloproteinase activity after acute myocardial infarction in pigs. Am J Physiol Heart Circ Physiol 2001;281:H987-H994

20. Sun Y, Zhang JQ, Zhang J, Lamparter S. Cardiac remodeling by fibrous tissue after infarction in rats. J Lab Clin Med 2000;135:316-23.

21. Gabbiani G, Hirschel BJ, Ryan GB, et al. Granulation tissue as a contractile organ. A study of structure and function. J Exp Med 1973;135:719-34.

22. Passier RC, Smits JF, Verluyten MJ, et al. Expression and localization of renin and angiotensinogen in rat heart after myocardial infarction. Am J Physiol 1996;271:H1040-H1048.
23. Sun Y, Zhang J, Zhang JQ, et al. Renin expression at sites of repair in the infarcted rat heart. J Mol Cell Cardiol 2001;33:995-1003.

24. Katwa LC, Campbell SE, Tyagi SC, et al. Cultured myofibroblasts generate angiotensin peptides de novo. J Mol Cell Cardiol 1997;29:1375-86.

25. Henkel DM. Glockner J, Miller WL. Association of myocardial fibrosis, B-type natriuretic peptide, and cardiac magnetic resonance parameters of remodeling in chronic ischemic cardiomyopathy. Am J Cardiol 2012;109:390-4.

26. Ismail TF, Prasad SK, Pennell DJ. Prognostic importance of late gadolinium enhancement cardiovascular magnetic resonance in cardiomyopathy. Heart 2012;98:438-42.

27. Ueland T, Aukrust P, Broch K, et al. Galectin-3 in heart failure: High levels are associated with all-cause mortality. Int J Cardiol 2011;150:361-4

28. Erkilet G, Özpeker C, Böthig D, et al. The biomarker plasma galectin-3 in advanced heart failure and survival with mechanical circulatory support device. J Heart Lung Transplant 2012;32:221-30.

29. Sato A, Aonuma K, Imanaka-Yoshida K, et al. Serum tenascin-C might be a novel predictor of left ventricular remodeling and prognosis after acute myocardial infarction. J Am Coll Cardiol 2006; 47:2319-25.

30. Sato M, Toyozaki T, Odaka K, et al. Detection of experimental autoimmune myocarditis in rats by $111 \mathrm{In}$ monoclonal antibody specific for tenascin-C. Circulation 2002;106:1397-402.

31. Seropian IM, Cerliani JP, Toldo S, et al. Galectin-1 controls cardiac inflammation and ventricular remodeling during acute myocardial infarction. Am J Pathol 2013;182:29-40. 